\title{
Cross-Calibration of POLAR: A Method to Correct the Detection Efficiencies in 1600 Pixels
}

\author{
Yuanhao Wang on behalf of the POLAR collaboration ${ }^{* *}$ \\ Key Laboratory for Particle Astrophysics, Institute of High Energy Physics \\ University of Chinese Academy of Sciences \\ E-mail: wangyuanhaodihep.ac.cn
}

\begin{abstract}
POLAR is a Compton polarimeter with 1600 pixels designed to measure the polarization of Gamma-Ray Bursts (GRBs). POLAR has been launched successfully onboard the Chinese space laboratory Tiangong-2 on 15th September 2016. The anisotropy of Compton scattering is used as the characteristic quantity to extract polarization degree and angle. The scattering angles are accumulated into a modulation curve and compared with simulated ones based on calibration data to determine the polarization. The differences of the detection efficiency in 1600 pixels between in-flight observation and simulation must be considered. Data from several bright GRBs are used for this purpose. We use the energy spectra of these GRBs provided by Fermi/GBM, KONUS/Wind and Swift to do simulations; the ground calibration data are used to characterize the performance of each individual pixel, including readout electronics. Comparing the simulation results with observation data, we obtain the detection efficiency of each of the 1600 pixels, which can then be corrected for measuring the polarization of these GRBs.
\end{abstract}

35th International Cosmic Ray Conference

10-20 July, 2017

Bexco, Busan, Korea

* Speaker.

${ }^{\dagger}$ A footnote may follow. 


\section{Introduction}

POLAR is a Compton polarimeter designed to measure the polarization of the prompt emission of GRBs. The instrument has been launched successfully on board the Chinese space laboratory Tiangong-2 on 15th September 2016. It consists 1600 pixels divided into 25 independent detector modules. Each detector module consists of 64 scintillator bars and is read out by a 64 channel

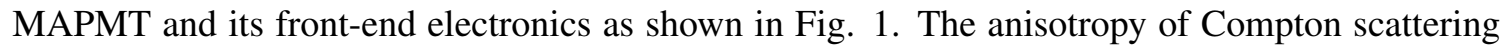
is used as the characteristic quantity of linear polarization of hard X-ray in $50 \mathrm{keV}-500 \mathrm{keV}$ energy range. The effective detection area for Compton scattering is about $80 \mathrm{~cm}^{2}$ and field of view is about $1 / 2$ of the full sky.
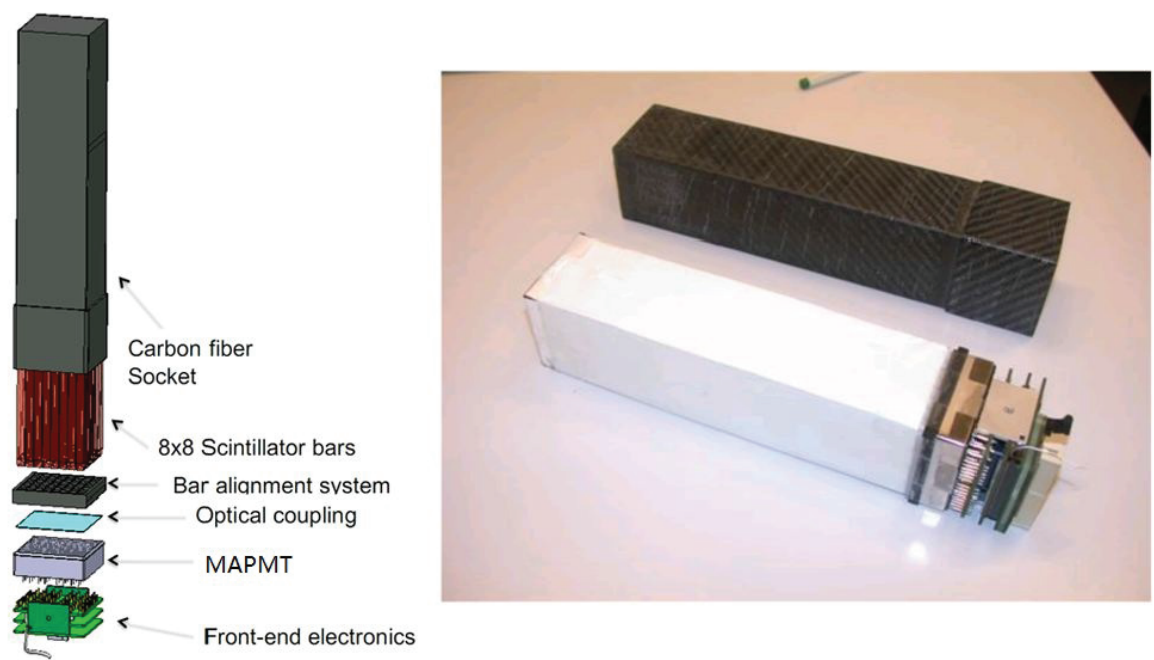

Figure 1: Left: the structure of one POLAR detector; Right: the photo of POLAR detector.

Photons in the $50-500 \mathrm{keV}$ energy range have a high probability of Compton scattering in the plastic scintillators. The incident photon takes the first Compton scattering in one plastic scintillator bar, and the recoil electrons deposit energy in the bar. The scattered photons generate a second Compton scattering or photoelectric absorption in another plastic scintillator bar to deposit energy until the scattered photons are completely absorbed or escape from the detectors. The Compton scattering is shown in Fig. [2. For the non-polarized photons, the azimuthal distribution of the photons after the scattering is isotropic, and for the polarized photons, the azimuthal distribution is anisotropic, as described in the Klein-Nishina equation [四:

$$
\frac{d \sigma}{d \Omega}=\frac{r_{0}^{2}}{2}\left(\frac{E^{\prime}}{E}\right)^{2}\left(\frac{E^{\prime}}{E}+\frac{E}{E^{\prime}}-2 \sin \theta^{2} \cos \eta^{2}\right)
$$

Here $r_{0}$ is the classical electron radius, $E$ and $E^{\prime}$ are the energy of the incident photon and the scattered photon, $\theta$ is the polar scattering angle and $\eta$ is the azimuthal scattering angle between the initial polarization vector and the direction of the scattered photon. Here the $\cos \varphi^{2}$ cause the anisotropic as the photons tend to be scattered perpendicular to the polarization vector. The distribution of azimuthal scattering angle $\varphi$, called modulation curve, can be described as: 


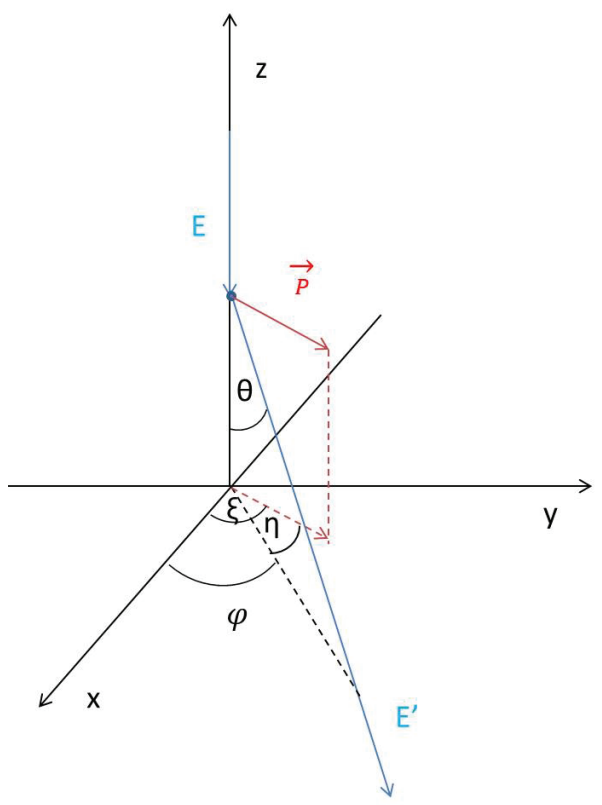

Figure 2: Compton scattering process. The incident photon is blue (Energy E) and polarization is red.

$$
f(\varphi)=\int \frac{d \sigma}{d \Omega} d \theta=k(1+\mu \cos (2 \varphi-2 \xi+\pi))
$$

Here $k$ is the normalization factor, $\xi$ is polarization angle, $\mu$ is the modulation factor which is proportional to the polarization degree. The polarization degree can be acquired from $\Pi=\mu / \mu_{100}$ where $\mu_{100}$ is the modulation factor for a $100 \%$ polarized beam [ [2], []]. We performed simulationd for $100 \%$ polarized beam to get the $\mu_{100}$.

Obviously, the most important systematic error in the polarization measurements is the difference between the instrument and simulator. In this paper, we build a simulator with 1600 pixels and select several bright GRBs to do the cross-calibration to get the difference of efficiency between simulations and observation.

\section{Sample selection}

Since launched on 15th September 2016, POLAR had detected at least 55 GRBs [ [4] during two periods: from $2016 / 09 / 21$ to $2016 / 10 / 14$ and from 2016/11/18 to 2017/04/01. We select a cross-calibration GRB sample based on these alternative conditions: bright (more than $10^{4}$ photons detected); confirmed by Fermi/GBM, Swift/BAT, SPI-ACS or Konus-Wind [G]; have accurate localization. At last, a sample of 8 GRBs was obtained to do cross-calibration. The basic information of these GRBs is listed in Table $\square$. 
Table 1: basic information of GRB sample

\begin{tabular}{lcccccc}
\hline Name & trigger time(UTC) & joint observation & T90(s) & total counts & RA(deg) & DEC(deg) \\
\hline GRB 161218B & 2016-12-18T08:32:41 & Fermi/GBM, KW & 26.30 & 29340 & 358.64 & -16.95 \\
GRB 161229A & 2016-12-29T21:03:49 & Fermi/GBM & 35.77 & 35134 & 81.03 & 6.31 \\
GRB 170114A & 2017-01-14T22:01:10 & Fermi/GBM & 8 & 26800 & 12.1 & -12.6 \\
GRB 170206A & 2017-02-06T10:51:57 & Fermi/GBM, KW & 1.2 & 12918 & 212.79 & 14.48 \\
GRB 170207A & 2017-02-07T21:45:04 & Fermi/GBM, IPN, KW & 39.47 & 63182 & 315.704 & 55.69 \\
GRB 170208C & 2017-02-08T13:16:33 & Fermi/GBM, SPI-ACS & 47.87 & 20354 & 284.42 & -0.11 \\
GRB 170210A & 2017-02-10T02:47:37 & Fermi/GBM, IPN, KW & 67.27 & 106099 & 226.55 & -65.1 \\
GRB 170306B & 2017-03-06T14:07:20 & Fermi/GBM, Swift-BAT, KW & 25 & 31640 & 154.65 & 51.63 \\
\hline
\end{tabular}

\section{Cross-calibration}

\subsection{Instrument response}

\subsubsection{Threshold}

Precise thresholds in all 1600 pixels of POLAR is crucial since the trigger is threshold dependent and simulation also needs exact threshold consistent with POLAR. Since voltage value (Vthr) and offset were changed several times in orbit, the real thresholds need to be recalculated for each GRB. The thresholds are fitted by error function:

$$
f(x)=\frac{2}{\sqrt{\pi}} \int_{0}^{\frac{x-p_{0}}{\sqrt{2} \cdot p_{1}}} e^{-t^{2}} d t
$$

Where $p_{0}$ is the threshold and $p_{1}$ is the error. Fig. [3 shows a typical result on one pixel.

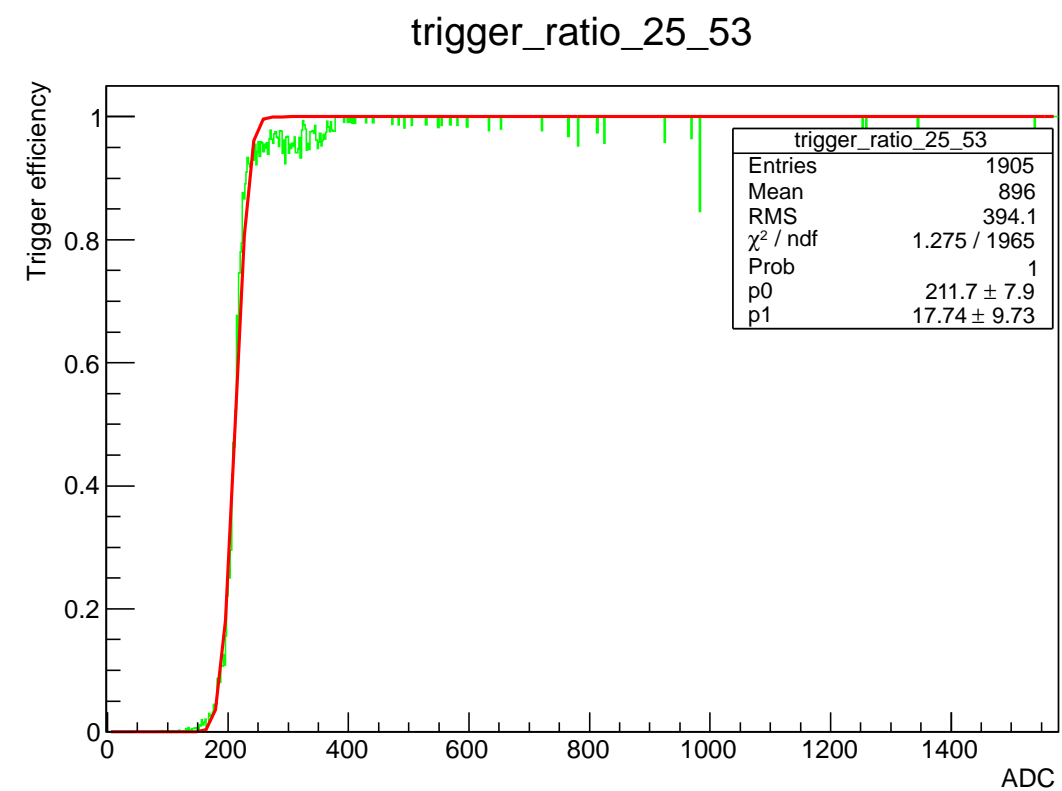

Figure 3: Threshold edge fit by error function. The green line is the observation data and the red one is the best fit line 


\subsubsection{Reconstruction of energy deposition}

Each of the 64 scintillator bars in the 25 detector modules is coupled to an anodes of a MAPMT. Channels in one module use the same high voltage value (HV). The Calibration of HVGain relation was performed by the ground experiment using the ${ }^{137} C s$ radioactive source [G]. The in-orbit HV value is used to calculate the gain for each GRB. Fig. 团 shows the gain of 1600 pixels for GRB 161218B.

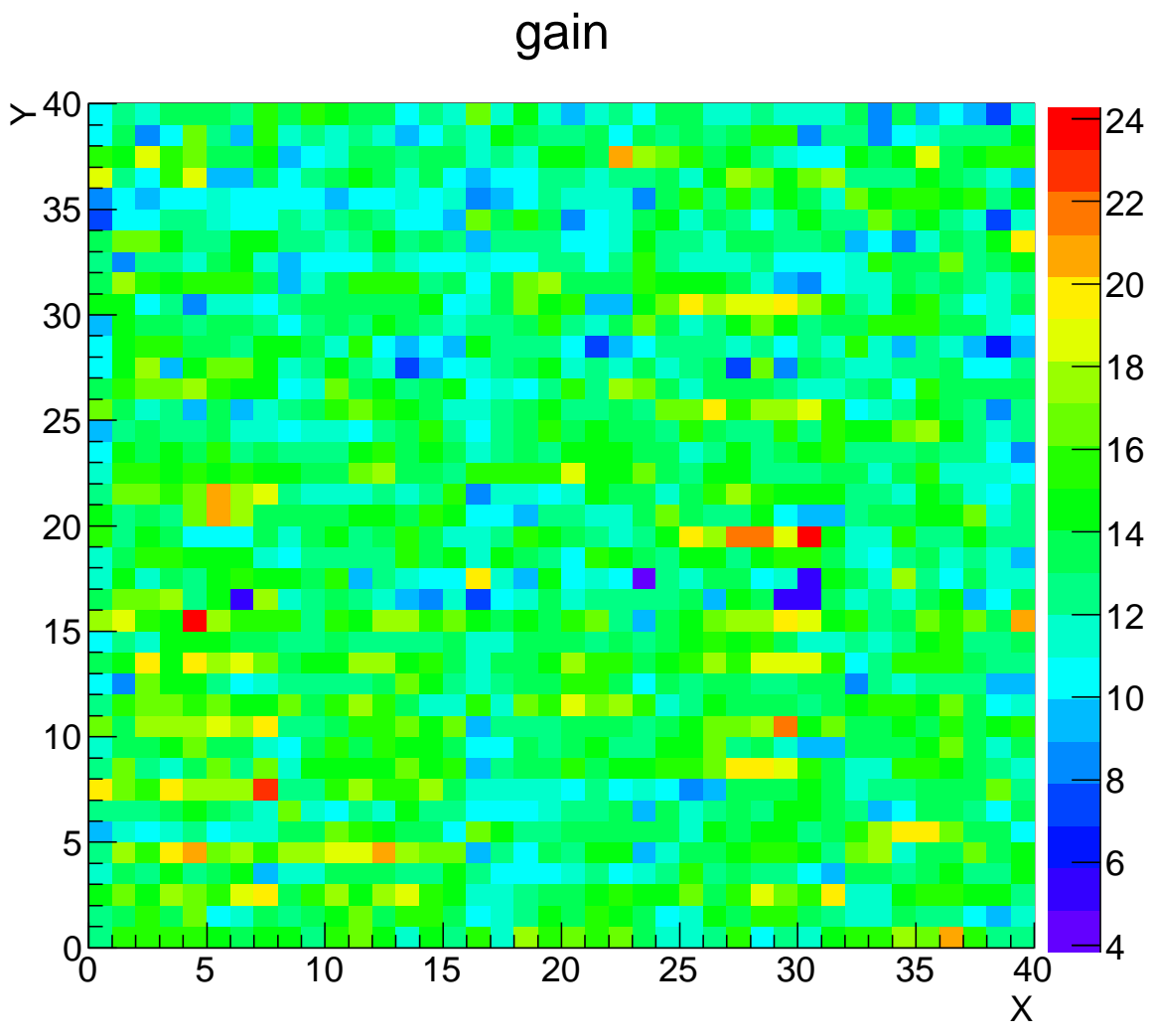

Figure 4: Distribution of gains in 1600 pixels. The unit of $\mathrm{z}$-axis is $\mathrm{ADC} / \mathrm{keV}$

\subsection{Data analysis}

Based on the threshold efficiency and gain, the energy range of each pixel was obtained, as shown in Fig. 1. Since the electronic noise may induce signals in the energy range of $0-10 \mathrm{keV}$, the bars with threshold values lower than $10 \mathrm{keV}$ were assumed to be $10 \mathrm{keV}$. The events with energy depositions below the threshold value were excluded. Then the trigger image of each GRB was generated, as shown in Fig 6 . The data just before and after each GRB trigger time and with a quiet performance in light curve was selected as the background, with a duration at least 10 times the GRB duration to ensure a small statistical error.

\subsection{Simulation}

A simulator of POLAR was produced using GEANT4.9.6. The gain and threshold efficiency obtained before was used in the simulations. Based on the GRB information, as well as incident 


\section{threshold}

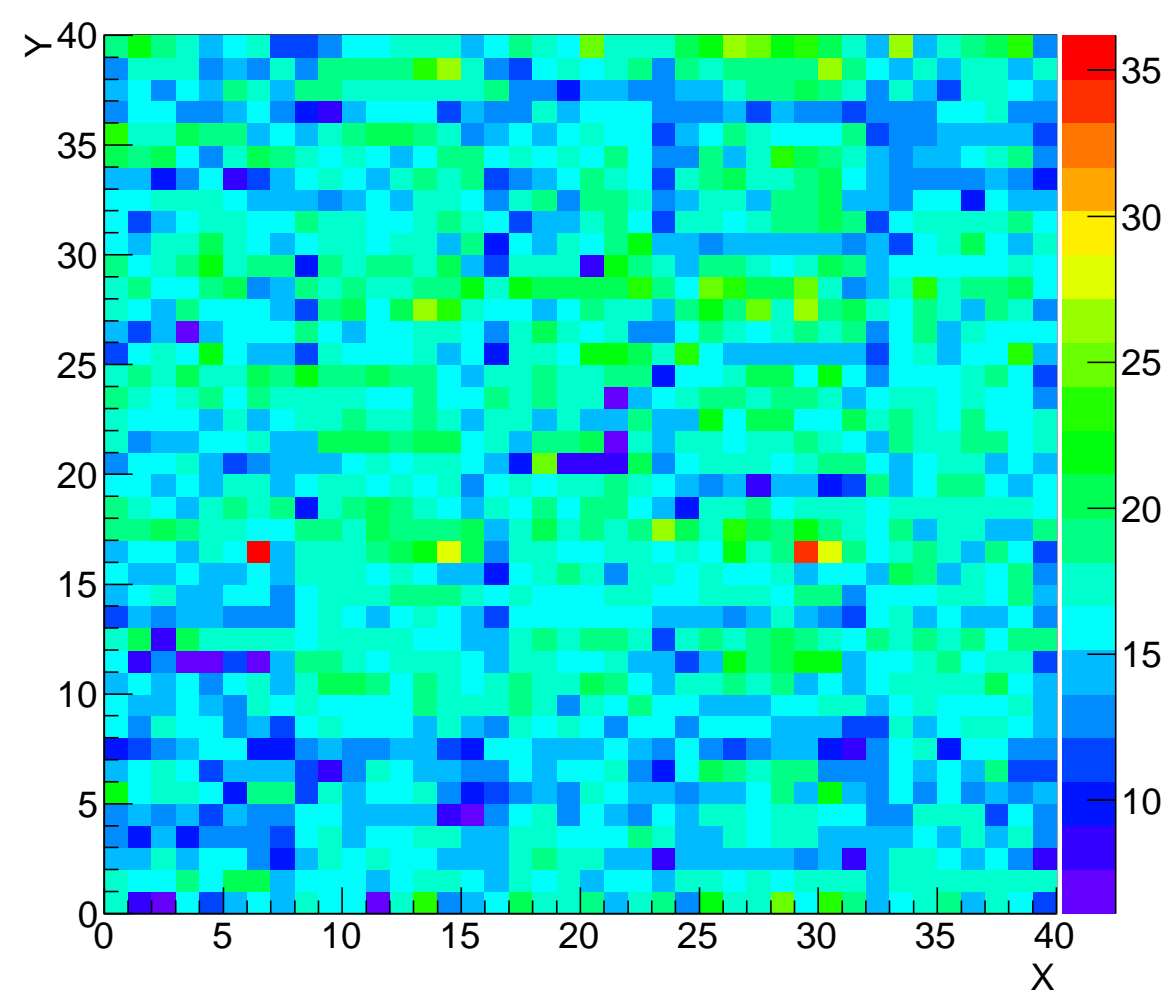

Figure 5: Distribution of thresholds in 1600 pixels, The unit of z-axis is keV.
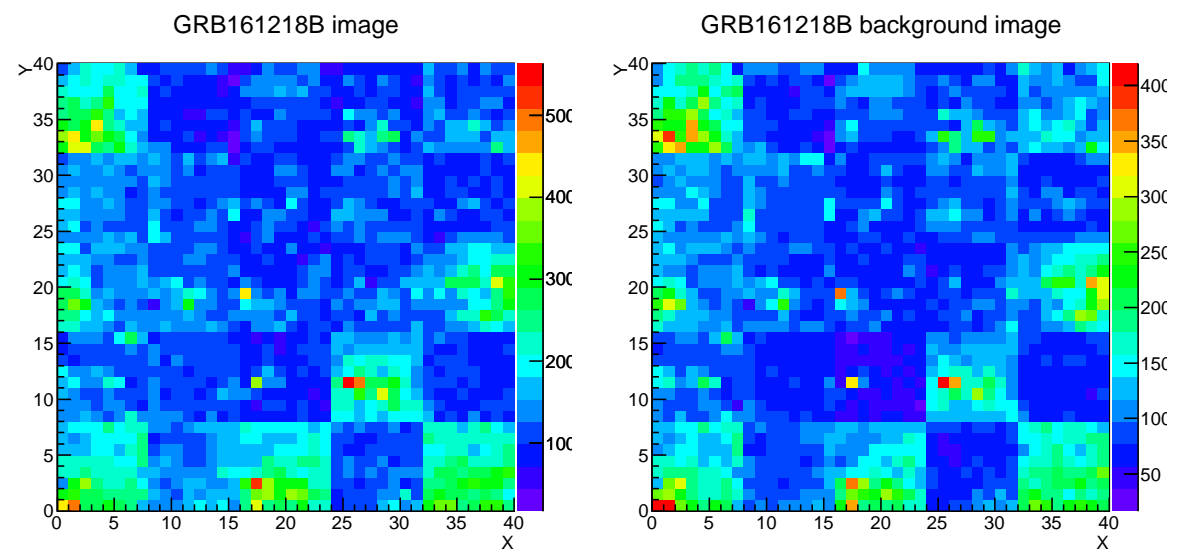

Figure 6: Left: 161218b trigger image. Right: the background trigger image. The unit of z-axis is counts. 
angle and spectrum, simulations were carried out for all GRBs. The data from the simulations was analyzed in the same way the POLAR data, the image of each GRB was generated.

\subsection{Non-uniformity efficiency}

Comparing the simulation image with observation data, we obtain the detection efficiency of each of the 1600 pixels.

$$
\text { Efficiency }[i]=\frac{\operatorname{Obs}[i]-B k g[i]}{\operatorname{Sim}[i]}
$$

The average efficiency of 1600 pixels is about 1 . Fig. $\square$ shows the result calculated only for one GRB, Fig. 8 shows the results for all the GRBs.
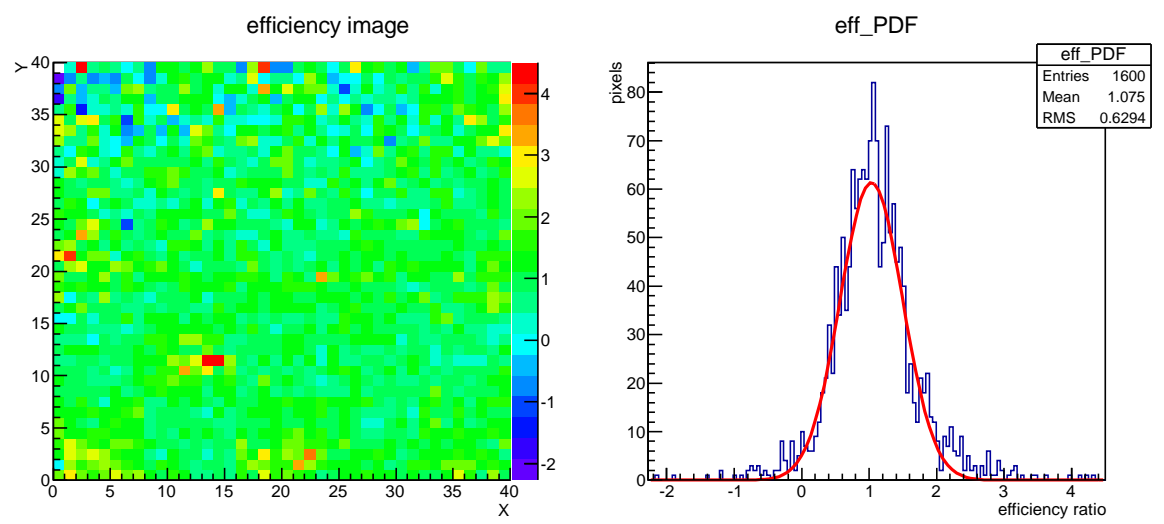

Figure 7: Left: Efficiency ratio between observation and simulation only for GRB 161218B, Right: The distribution of efficiency.
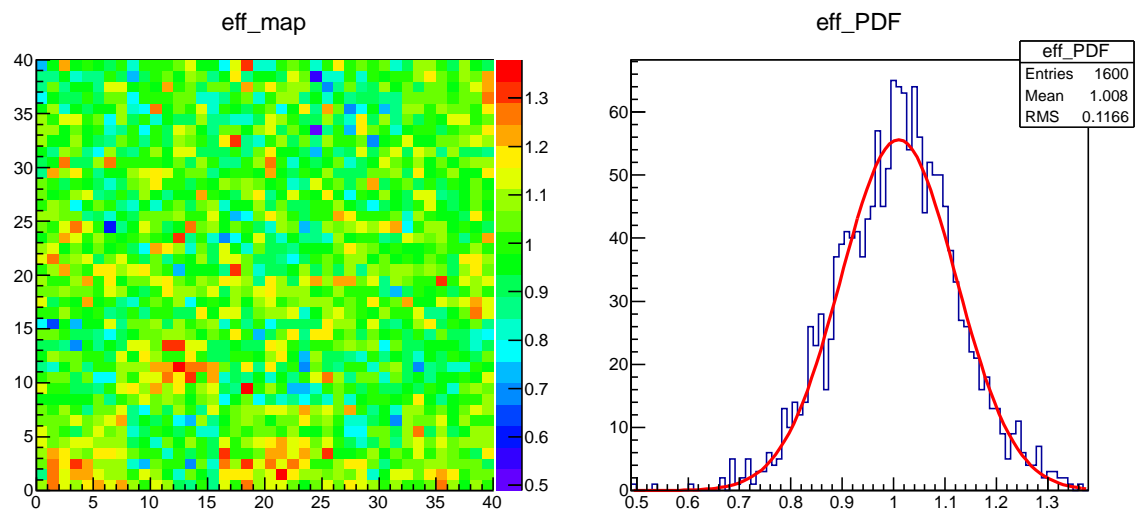

Figure 8: Efficiency ratio between observation and simulation for 8 GRBs, Right: The distribution of efficiency.

\section{Conclusion}

In order to eliminate the systematic error, we analyzed the differences of the detection efficiency in 1600 pixels between in-orbit observation and simulation. The sample consists 8 bright GRBs 
were used to do this work. We use the energy spectra of these GRBs provided by Fermi/GBM, KONUS/Wind and Swift and used the localization provided by Fermi/GBM and IPN triangulation to do simulations; the ground calibration data are used to characterize the performance of each individual pixel. Comparing the simulation image with observation data, we obtained the detection efficiency of each of the 1600 pixels, this can be used to correct the data for polarization measurement of GRBs.

\section{References}

[1] O. Klein, Y. Nishina, Z. Phys. 52(1929)853

[2] N. Produit ,et al., Nucl. Instr. Meth. Phys. Res. A, 550 (2005) 616-625

[3] S. Orsi,et al., Nucl. Instr. Meth. Phys. Res. A, 648 (1) (2011) 139-154

[4] Xiong S.L. et al., ICRC Conf. Proc.2017

[5] https://gcn.gsfc.nasa.gov/gen3_archive.html

[6] X.F. Zhang, W. Hajdas et al., Optimization of the gain factors and parameter settings for the new gamma-ray burst polarimeter POLAR, submitted to Nucl. Instr. Meth. Phys. Res. A

[7] M. Kole et al., ICRC Conf. Proc. 2017

[8] M. Kole, et al., submitted to Nucl. Instr. and Meth. A 